\title{
Generalized Self-Consistent Model for Predicting Thermal Conductivity of Composites with Aligned Short Fibers
}

\author{
Jae-Kon Lee and Jin-Gon Kim \\ School of Mechanical and Automotive Engineering, Catholic University of Daegu, Gyeongsan, Gyeongbuk 712-702, Korea
}

Thermal conductivities of aligned short fiber composites in the longitudinal and transverse directions are derived by extending the generalized self-consistent model proposed by Benveniste and Miloh. Their final forms are semi-explicitly expressed as functions of constituents' thermal conductivities, fiber aspect ratio, and the aspect ratio of the matrix surrounding the fiber. The results are proved to be consistent with those of composites with extreme shapes of fillers such as sphere and continuous fiber by simply changing a confocality variable. The present results for aligned short fiber composites are compared with Wu's upper and lower bounds to be validated. Finally, the predictions by the present model and modified Eshelby model are compared in terms of the thermal conductivity ratio of constituents, fiber aspect ratio, and fiber volume fraction and their accuracy for the predictions are evaluated by the experimental results from the literature. [doi:10.2320/matertrans.M2010249]

(Received July 26, 2010; Accepted August 20, 2010; Published October 25, 2010)

Keywords: composite material, thermal conductivity, generalized self-consistent model, Eshelby model, Mori-Tanaka's mean field approach

\section{Introduction}

Micromechanics models such as self-consistent model (SCM), generalized self-consistent model (GSCM), differential scheme, and modified Eshelby's model (MEM) have been employed for predicting the thermo-mechanical properties of composites. Hill and Budiansky have applied SCM to a composite reinforced with spherical fillers, which determines the elastic constants of the composite by embedding only one filler into an infinite domain with the composite property determined. ${ }^{1,2)}$ On the other hand, MEM, the original Eshelby model combined with Mori-Tanaka's mean field approach, has been widely employed for predicting the thermo-mechanical properties of composites reinforced with various shapes of fillers. ${ }^{3-9)}$ Hatta and Taya derived the thermal conductivity of a composite with short fibers using both MEM and the analogy between the elasticity and the steady-state heat conduction. ${ }^{7)}$ It has been applied to composites with various shapes of fillers and widely used due to its simplicity of a computation. ${ }^{8)}$

GSCM, closely related with SCM, has been proposed by Kerner, which is extended by Christen and Lo and called a "three-phase model". ${ }^{10,11)}$ GSCM predicts the thermo-mechanical properties of composites by embedding a filler and surrounding matrix shell into the infinite domain of the unknown properties of the composite. Although its procedures for a derivation and computation are rather complicated, Christensen ${ }^{12)}$ evaluated the theoretical models for the calculation of the overall elastic constants of composites and concluded that only GSCM gives physically reasonable results at high concentrations of fillers. So, it seems to be a more appropriate tool for a homogenization process. ${ }^{12,13)}$ This model has been applied to composites reinforced with simple shapes such as spherical fillers and continuous fibers for predicting the thermal conductivity of the composites, where a contact resistance at the interface between a matrix and fillers is considered. ${ }^{14-16)} \mathrm{A}$ research on the thermal conductivity of composites with short fiber using GSCM is very limited. GSCM is presented for particulate composites, but particular emphasis is given to the special case of a cracked body with randomly oriented elliptical cracks. ${ }^{17)}$ The thermal conductivity of composites with randomly oriented spheroidal fiber has been computed for the fiber aspect ratio of 10 and compared with the upper and lower bounds of Hahin and Shtrikman.

In this study, the thermal conductivities of aligned short fiber composites in the longitudinal and transverse directions are derived by extending GSCM proposed by Miloh and Benveniste $^{17)}$ and the results are expressed semi-explicitly for convenience of later use. The present results are compared with those of composites with the extreme shapes of fillers such as spherical filler and continuous fiber by simply changing the value of a confocality variable. The results of short fiber composites by GSCM are validated by comparing with the narrow bounds proposed by $\mathrm{Wu},{ }^{18)}$ which are compared with $\mathrm{MEM}^{12)}$ and the experimental results ${ }^{19)}$ and fully discussed.

\section{GSCM for Predicting Thermal Conductivity of Composites}

GSCM is conceptually explained here and its detailed derivations are given in the literatures. ${ }^{14-17)}$ In SCM, the thermal conductivity of a composite is derived by embedding one filler into an infinite matrix having the average properties of the composite. GSCM employs the same embedding process as SCM, but the embedded filler is different. It consists of two confocal ellipsoids. The inner ellipsoid is the fiber and outer one is the matrix. The fiber volume fraction in the embedded filler is the same as the one in the entire composite. The embedded filler is regarded as the composite of the same material properties as the composite under investigation. By applying the Laplace equation and boundary conditions to the infinite composite domain, the temperature distribution in the whole domain is determined analytically, based on which the thermal conductivity of the composite is defined as the ratio of the average heat flux to the average temperature gradient in the embedded filler. 


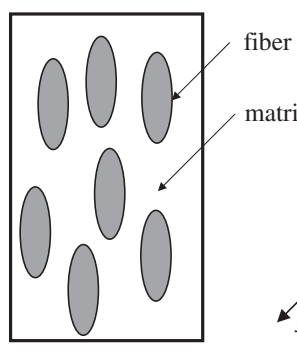

(a)

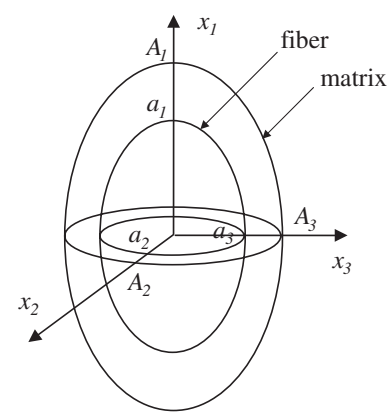

(b)
Fig. 1 (a) Composite with aligned short fibers and (b) dimensions of two confocal prolate spheroids representing fiber and matrix in the rectangular coordinate system.

Based on the aforementioned concept, Benveniste and Miloh ${ }^{17)}$ derived the thermal conductivities of composites with randomly oriented short fibers and their derivations are summarized here. As shown in Fig. 1, the embedded filler is represented by two confocal ellipsoids, where the semi-axes of the inner and outer surfaces are given by $a_{i}$ and $A_{i}, i=1,2$, 3 , respectively. The thermal conductivity of the embedded filler, $k_{i}^{\mathrm{c}}$, is given by

$$
\frac{k_{i}^{\mathrm{c}}}{k_{\mathrm{m}}}=1+\frac{W_{1}^{(i)}\left(A_{1}, A_{1}\right)}{\frac{k_{\mathrm{f}}}{k_{\mathrm{f}}-k_{\mathrm{m}}} W_{1}^{(i)}\left(a_{1}, a_{1}\right)-W_{1}^{(i)}\left(A_{1}, a_{1}\right)},
$$

where $k$ represents thermal conductivity and subscripts $\mathrm{m}, \mathrm{f}$, $i$, and superscript $\mathrm{c}$ do the matrix, filler, the direction, and composite, respectively. $W_{1}^{(i)}(r, s)$ denotes pseudo-Wronskian and is defined as

$$
W_{1}^{(i)}(r, s)=\frac{F_{1}^{(i)}(r)}{E_{1}^{(i)}(r)}-\frac{\dot{F}_{1}^{(i)}(s)}{\dot{E}_{1}^{(i)}(s)},
$$

where $E_{1}^{(i)}$ and $F_{1}^{(i)}$ denote the Lame functions of the first and second kind of order one and of type $i$, respectively and the dot denotes differentiation with respect to the argument. $\alpha$ and $\beta$ imply the confocality of the ellipsoids:

$$
\begin{aligned}
\alpha^{2} & =a_{1}^{2}-a_{3}^{2}=A_{1}^{2}-A_{3}^{2}, \\
\beta^{2} & =a_{1}^{2}-a_{2}^{2}=A_{1}^{2}-A_{2}^{2} .
\end{aligned}
$$

The shape of the ellipsoids is further simplified for derivations as $a_{2}=a_{3}$ and $A_{2}=A_{3}$, i.e., $\alpha=\beta$. The aspect ratio of the short fiber is defined as $c=a_{1} / a_{3}$.

From eq. (1), the thermal conductivities of the composite in the longitudinal and transverse directions, $k_{1}^{\mathrm{c}}$ and $k_{3}^{\mathrm{c}}$, can be expressed as

$$
\begin{aligned}
& \frac{k_{1}^{\mathrm{c}}}{k_{\mathrm{m}}}=1+\frac{W_{1}^{1}\left(A_{1}, A_{1}\right)}{\frac{k_{\mathrm{f}}}{k_{\mathrm{f}}-k_{\mathrm{m}}} W_{1}^{1}\left(a_{1}, a_{1}\right)-W_{1}^{1}\left(A_{1}, a_{1}\right)}, \\
& \frac{k_{3}^{\mathrm{c}}}{k_{\mathrm{m}}}=1+\frac{W_{1}^{2}\left(A_{1}, A_{1}\right)}{\frac{k_{\mathrm{f}}}{k_{\mathrm{f}}-k_{\mathrm{m}}} W_{1}^{2}\left(a_{1}, a_{1}\right)-W_{1}^{2}\left(A_{1}, a_{1}\right)} .
\end{aligned}
$$

By inserting pseudo-Wronskian and Lame functions into the final forms, eq. (4), the thermal conductivities of the composite in the longitudinal and transverse directions can be summarized as

$$
\begin{aligned}
& \frac{k_{1}^{\mathrm{c}}}{k_{\mathrm{m}}}=1+\frac{f}{\frac{k_{\mathrm{f}}}{k_{\mathrm{f}}-k_{\mathrm{m}}}-\frac{a_{1} a_{3}^{2}}{2 \alpha^{3}}\left[\ln \frac{\left(A_{1}+\alpha\right)\left(a_{1}-\alpha\right)}{\left(A_{1}-\alpha\right)\left(a_{1}+\alpha\right)}-2 \alpha\left(\frac{1}{A_{1}}-\frac{1}{a_{1}}\right)\right]-1},
\end{aligned}
$$

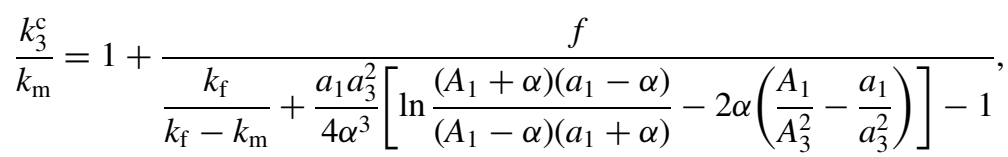

where the fiber volume fraction $f$ is defined as follows:

$$
f=\frac{a_{1} a_{3}^{2}}{A_{1} A_{3}^{2}} .
$$

As shown in eq. (5), the thermal conductivities of the composite with aligned short fibers are expressed as the simple forms, which are further simplified in the Section 3.

\section{Results and Discussion}

The results for the short fiber composite are extended to composites with other shapes of fillers such as the sphere and continuous fiber by simply changing the confocality variable, $\alpha$, in eq. (5). These results are compared with the results in the literatures.

\subsection{Composite with spherical fillers}

Spherical fillers are the one of extreme shapes of spheroids and are simulated by approaching $\alpha$ as zero. The thermal conductivities of the composite with spherical fillers in both directions are derived by eq. (5). By using Taylor series expansion, a logarithmic function $\ln (1+x)$ can be expressed by

$$
\ln (1+x)=\sum_{k=1}^{\infty}(-1)^{k+1} \frac{x^{k}}{k}
$$

The terms in the denominator of eq. (5a) can be expanded as

$$
\begin{gathered}
\frac{a_{1} a_{3}^{2}}{2 \alpha^{3}}\left[\ln \frac{\left(A_{1}+\alpha\right)\left(a_{1}-\alpha\right)}{\left(A_{1}-\alpha\right)\left(a_{1}+\alpha\right)}-2 \alpha\left(\frac{1}{A_{1}}-\frac{1}{a_{1}}\right)\right] \\
=\frac{a_{1} a_{3}^{2}}{\alpha^{3}} \sum_{k=2}^{\infty} \frac{\alpha^{2 k-1}}{2 k-1}\left(\frac{1}{A_{1}^{2 k-1}}-\frac{1}{a_{1}^{2 k-1}}\right) .
\end{gathered}
$$

As $\alpha$ becomes zero, eq. (8) is simplified as

$$
\lim _{\alpha \rightarrow 0} \frac{a_{1} a_{3}^{2}}{\alpha^{3}} \sum_{k=2}^{\infty} \frac{\alpha^{2 k-1}}{2 k-1}\left(\frac{1}{A_{1}^{2 k-1}}-\frac{1}{a_{1}^{2 k-1}}\right)=\frac{1}{3}(f-1) .
$$

Finally, the thermal conductivity of the composite in the longitudinal direction can be finally reduced to 


$$
\frac{k_{1}^{\mathrm{c}}}{k_{\mathrm{m}}}=\frac{2(1-f) k_{\mathrm{m}}+(1+2 f) k_{\mathrm{f}}}{(2+f) k_{\mathrm{m}}+(1-f) k_{\mathrm{f}}},
$$

which is exactly the same result obtained in literatures. ${ }^{14-16)}$

Following the same procedures in the above derivations, the thermal conductivity of composite in the transverse direction, eq. (5b), can be derived. As $\alpha$ approaches zero, the terms in the denominator of eq. (5a) can be reduced to

$$
\begin{aligned}
& \lim _{\alpha \rightarrow 0} \frac{a_{1} a_{3}^{2}}{4 \alpha^{3}}\left[\ln \frac{\left(A_{1}+\alpha\right)\left(a_{1}-\alpha\right)}{\left(A_{1}-\alpha\right)\left(a_{1}+\alpha\right)}-2 \alpha\left(\frac{A_{1}}{A_{3}^{2}}-\frac{a_{1}}{a_{3}^{2}}\right)\right] \\
& =-\frac{1}{3}(f-1) .
\end{aligned}
$$

The thermal conductivity of the composite in the transverse direction is derived as

$$
\frac{k_{3}^{\mathrm{c}}}{k_{\mathrm{m}}}=\frac{2(1-f) k_{\mathrm{m}}+(1+2 f) k_{\mathrm{f}}}{(2+f) k_{\mathrm{m}}+(1-f) k_{\mathrm{f}}},
$$

which is equivalent to eq. (10).

\subsection{Composite with continuous fillers}

The results for the short fiber composite can be extended to continuous fiber composite by taking $\alpha$ as infinity. By using the relation from eq. (3), $A_{1}-\alpha=A_{3}^{2} /\left(A_{1}+\alpha\right)$, the term in the bracket of eq. (5a) can be simplified as

$$
\begin{aligned}
\lim _{\alpha \rightarrow \infty} \frac{a_{1} a_{3}^{2}}{2 \alpha^{3}} \ln \frac{\left(A_{1}+\alpha\right)\left(a_{1}-\alpha\right)}{\left(A_{1}-\alpha\right)\left(a_{1}+\alpha\right)} \\
=\lim _{\alpha \rightarrow \infty} \frac{a_{1} a_{3}^{2}}{4 \alpha^{3}} \ln \frac{a_{3}^{2}\left(A_{1}+\alpha\right)^{2}}{A_{3}^{2}\left(a_{1}+\alpha\right)^{2}}=0 .
\end{aligned}
$$

The second term in the bracket becomes zero:

$$
\lim _{\alpha \rightarrow \infty} \frac{a_{1} a_{3}^{2}}{\alpha^{2}}\left(\frac{1}{A_{1}}-\frac{1}{a_{1}}\right)=0 .
$$

By inserting eqs. (13) and (14) into eq. (5a), the thermal conductivity of the composite in the longitudinal direction can be summarized as

$$
k_{1}^{\mathrm{c}}=(1-f) k_{\mathrm{m}}+f k_{\mathrm{f}},
$$

which is equivalent to the result by the parallel model.

By using eq. (13), the first term in the bracket of eq. (5b) becomes zero. The second term in the bracket of eq. (5b) can be changed as

$$
\lim _{\alpha \rightarrow \infty} \frac{a_{1} a_{3}^{2}}{2 \alpha^{2}}\left(\frac{A_{1}}{A_{3}^{2}}-\frac{a_{1}}{a_{3}^{2}}\right)=\frac{1}{2}(f-1) .
$$

The thermal conductivity of the composite in the transverse direction is simplified as

$$
\frac{k_{3}^{\mathrm{c}}}{k_{\mathrm{m}}}=\frac{(1-f) k_{\mathrm{m}}+(1+f) k_{\mathrm{f}}}{(1+f) k_{\mathrm{m}}+(1-f) k_{\mathrm{f}}},
$$

which is the same result derived by GSCM under the same condition. ${ }^{14,16)}$

\subsection{Composite with short fibers}

Equation (3) gives the following relations:

$$
\frac{\alpha}{a_{1}}=\sqrt{1-1 / c^{2}}, \quad \frac{\alpha}{A_{1}}=\sqrt{1-1 / \xi^{2}},
$$

where $\xi$ represents the aspect ratio of the outer ellipsoid or the aspect ratio of the matrix surrounding the fiber and is defined as

$$
\xi=\frac{A_{1}}{A_{2}}
$$

By inserting eq. (18) into eq. (5) and rearranging them, the thermal conductivities of the composite in both directions can be finally simplified as

$$
\begin{aligned}
\frac{k_{1}^{\mathrm{c}}}{k_{\mathrm{m}}} & =1+\frac{\frac{f}{k_{\mathrm{f}}-k_{\mathrm{m}}}-\frac{c}{\left(c^{2}-1\right)^{3 / 2}} \ln \left[\frac{\left(\xi+\sqrt{\xi^{2}-1}\right)\left(c-\sqrt{c^{2}-1}\right)}{e^{\sqrt{\xi^{2}-1} / \xi-\sqrt{c^{2}-1} / c}}\right]}{\frac{f}{k_{\mathrm{m}}^{\mathrm{c}}}}=1+\frac{\frac{k_{\mathrm{m}}}{k_{\mathrm{f}}-k_{\mathrm{m}}}-\frac{c}{2\left(c^{2}-1\right)^{3 / 2}} \ln \left[\frac{\left(\xi+\sqrt{\xi^{2}-1}\right)\left(c-\sqrt{c^{2}-1}\right)}{e^{\xi \sqrt{\xi^{2}-1}-c \sqrt{c^{2}-1}}}\right]}{} .
\end{aligned}
$$

Once $\xi$ is computed for given $c$ and $f$, the thermal conductivities of the composite can be determined by eqs. (20). $\xi, c$, and $f$ are related through the fiber volume fraction and their relationship is given by

$$
f=\frac{a_{1}^{3} \xi^{2}}{A_{1}^{3} c^{2}},
$$

which is determined by the confocality and fiber volume fraction.

By using eqs. (18) and (21), the nonlinear equation including $\xi, f$, and $c$ is derived as

$$
\xi^{6}-3 \xi^{4}+\left[3-\left(c^{2}-1\right)^{3}(f / c)^{2}\right] \xi^{2}-1=0 .
$$

Solving eq. (22) numerically, the thermal conductivities of the composite are computed from eq. (20).
Researches on upper and lower bounds of thermal conductivity for composites with spherical or continuous fillers have been rigorously made, which have been used to validate a new model. These researches for composites with aligned short fibers are limited, so the validity of the present model is investigated by the model newly proposed by $\mathrm{Wu}{ }^{18)}$ He recommended the geometric parameters for the prediction of upper and lower bounds to be between 3 and 5 and demonstrated that the upper bound decreases and the lower bound increases with the decreasing of these parameters, resulting in narrower bounds. The longitudinal and transverse thermal conductivities predicted by the present model are compared with Wu's narrow bounds with geometric parameters of 3, which are shown in Fig. 2 as a function of the fiber volume fraction. The effects of thermal conductivity ratio 
(a)

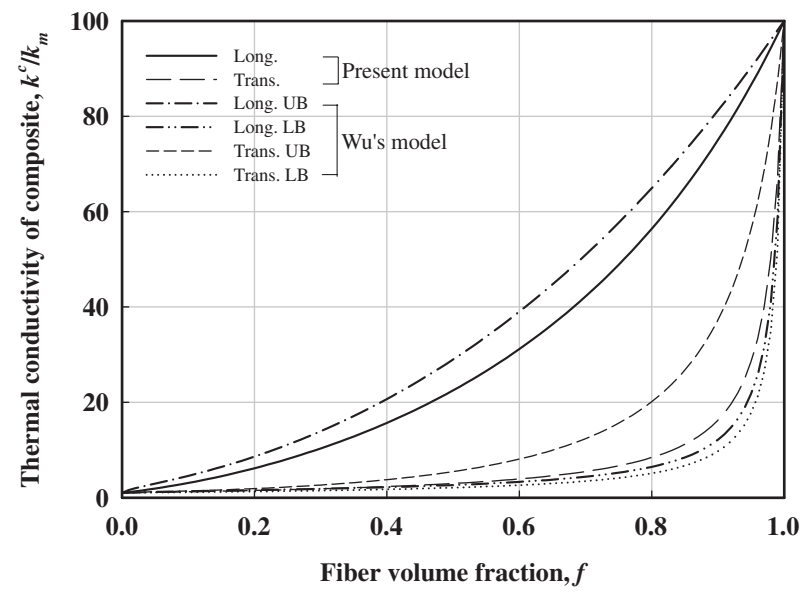

(c)

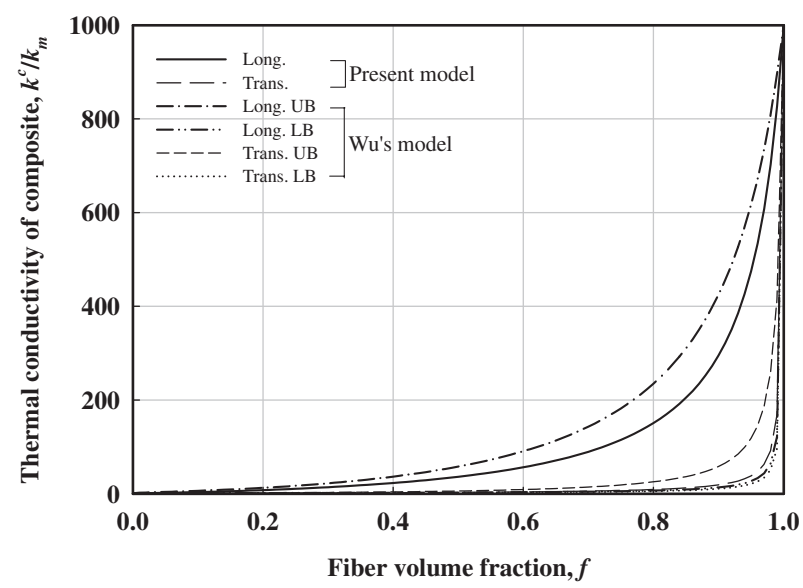

(b)

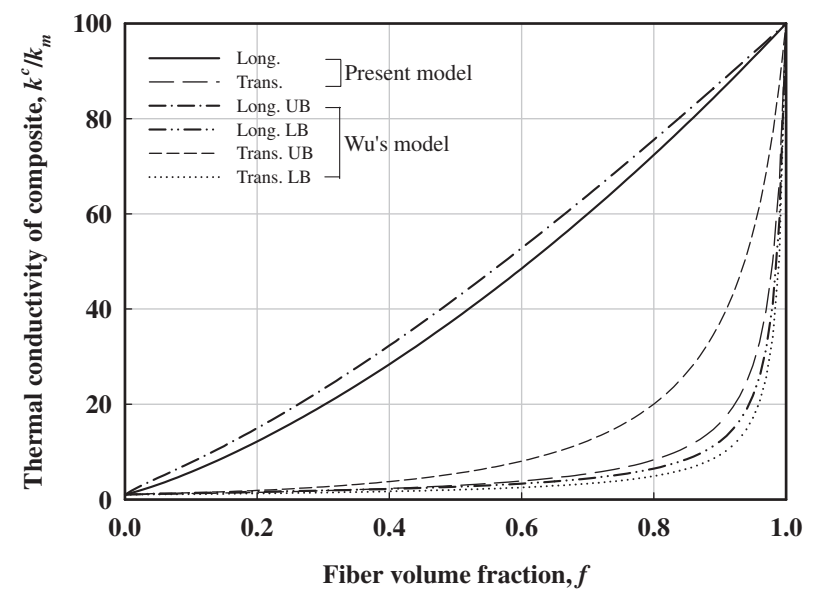

(d)

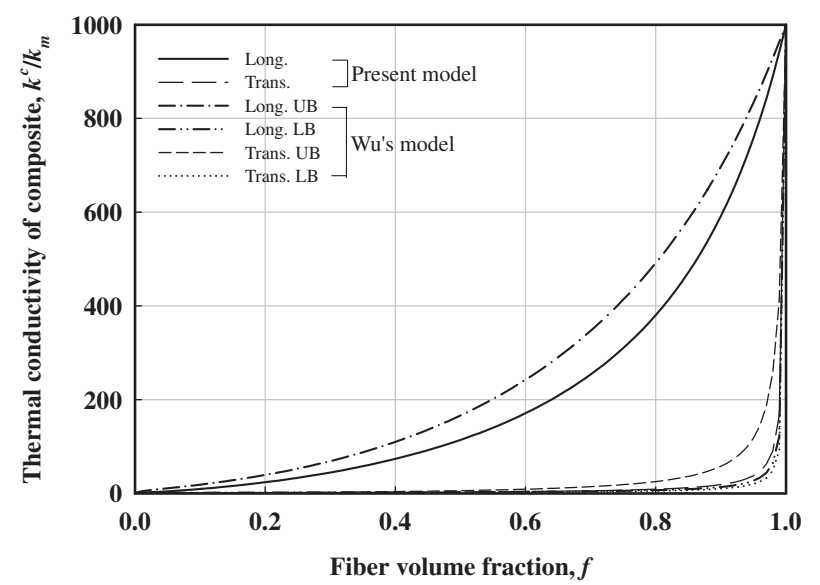

Fig. 2 Comparison of the predictions of thermal conductivities of aligned short fiber composites by the present model with Wu's upper (UB) and lower (LB) bounds; (a) $k_{\mathrm{f}} / k_{\mathrm{m}}=100, c=5$, (b) $k_{\mathrm{f}} / k_{\mathrm{m}}=100, c=10$, (c) $k_{\mathrm{f}} / k_{\mathrm{m}}=1000, c=5$, and (d) $k_{\mathrm{f}} / k_{\mathrm{m}}=1000, c=10$.

$\left(k_{\mathrm{f}} / k_{\mathrm{m}}\right)$ and fiber aspect ratio $(c)$ on the predicted results are also included. The thermal conductivity ratios are 100 and 1000 , and the fiber aspect ratios are 5 and 10. It can be concluded from the comparisons that the results predicted by the present model fall within these bounds for all fiber volume fractions, thermal conductivity ratios, and fiber aspect ratios under investigation.

As shown in Fig. 3, the thermal conductivities of the composite with aligned short fibers in both directions predicted by the present model are compared with the results by $\mathrm{MEM}^{7)}$ as a function of fiber aspect ratio, thermal conductivity ratio, and fiber volume fraction, where the results by MEM are normalized by those by the present model. The transverse thermal conductivities predicted by both models reveal no significant difference for all the variables investigated. MEM overestimates slightly the transverse thermal conductivity with increasing the thermal conductivity ratio and decreasing the fiber aspect ratio, comparing with the present model. However, the longitudinal thermal conductivities by both models show large discrepancies. The results by MEM are always smaller than those by the present model and their differences get larger with increasing the thermal conductivity ratio. The fiber aspect ratios at which maximum differences between them occur depend on the thermal conductivity ratios. According to Fig. 3, the maximum differences for the thermal conductivity ratios of 10,100 , and 1000 take places at the fiber aspect ratios of $2.25,5$, and 10 , respectively. Based on the parametric studies and Sections 3.1 and 3.2, the effect of fiber aspect ratio on the predictions by the two models can be summarized. The predictions by the two models are the same at the fiber aspect ratio of 1 . As the fiber aspect ratio increases, the differences reach maximum and decline with further increasing the fiber aspect ratio. Finally, they disappear at the fiber aspect ratio of infinity.

The predicted trends as a function of the fiber volume fraction by the two models are rather complicated. In general, their differences increase as the fiber volume fraction increases, and decrease with further increasing it. But the trends are different, depending on the thermal conductivity ratio and fiber aspect ratio. The maximum differences take place at around $f=0.6$ for the thermal conductivity ratios and the corresponding fiber aspect ratio for them increases with increasing the thermal conductivity ratio.

Next, the predictions by the two models are compared with the experimental results from the literature. ${ }^{19)}$ The model 

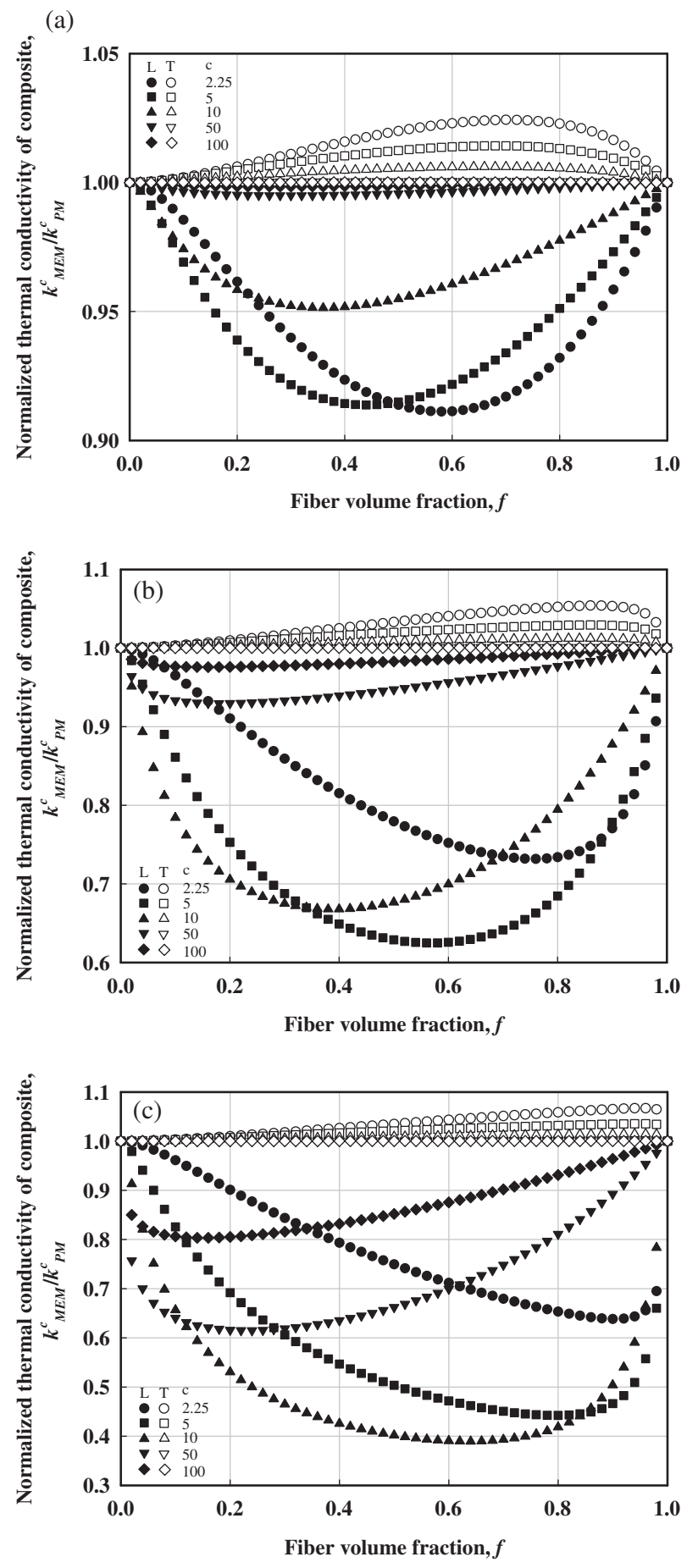

Fig. 3 The thermal conductivities by modified Eshelby model (MEM) are normalized by those by the present model (PM) for the aligned short fiber composite and $\mathrm{L}, \mathrm{T}$, and $\mathrm{c}$ stand for the longitudinal and transverse directions, and fiber aspect ratio, respectively; (a) $k_{\mathrm{f}} / k_{\mathrm{m}}=10$, (b) $k_{\mathrm{f}} / k_{\mathrm{m}}=100$, and (c) $k_{\mathrm{f}} / k_{\mathrm{m}}=1000$.

composite is a polymer matrix reinforced with aluminum nitride and the thermal conductivities of the matrix and filler are 0.195 and $220 \mathrm{~W} / \mathrm{mK}$, respectively. The shape of the filler is not perfect sphere, so the filler can be treated as the short fiber whose average aspect ratio is assumed to be $2.25 .^{20)}$ The fillers are assumed to be aligned in the longitudinal direction. The thermal conductivities of the composite in the longitudinal direction by the two models are computed with the thermal conductivity ratio of 1,128 , which

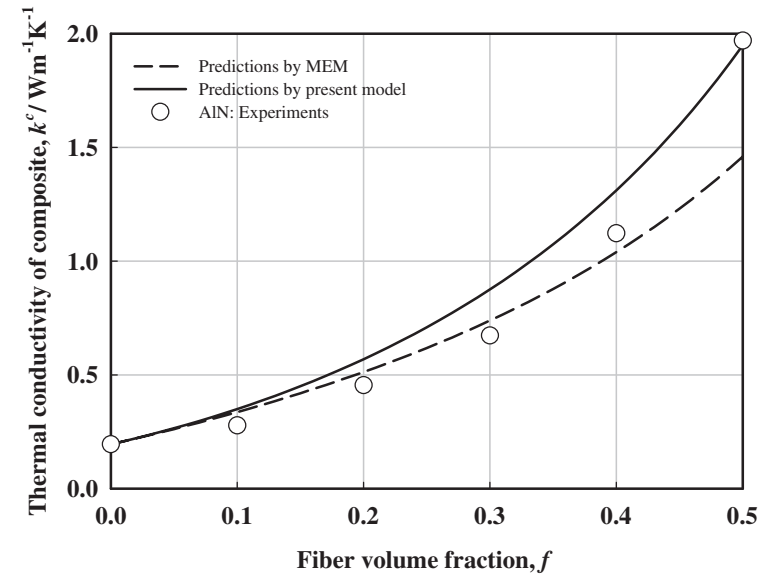

Fig. 4 Comparison of the experimental results ${ }^{19)}$ with the predicted results by the modified Eshelby model (MEM) and the present model for a polymer matrix with aluminum nitride.

are compared with the experimental results. As shown in Fig. 4, MEM predicts the same thermal conductivity as the experimental results except for $f=0.5$, while the present model does a little bit higher than the experimental results except for $f=0.5$. It has been shown by Hatta and Taya ${ }^{9)}$ that the thermal conductivities of the aligned and completely random short fiber composites give the maximum and minimum values for given $f$, and the latter is about $80 \%$ of the former for the composite with the fiber aspect ratio of 2 and $f=0.4$. Since the fillers are randomly oriented in the model composite, both models naturally overestimate the thermal conductivity in some extent. Once the filler orientation is considered, the predictions by both models tend to be lower than the results for the aligned short fiber composite. The predictions by the two models would be in relatively good agreement with the experimental results for $f$ of 0.3 or smaller. However, the present model would predict better than MEM by applying the result by Hatta and Taya ${ }^{9)}$ for $f$ of 0.4 or higher. It can be reached from the parametric studies and Fig. 4 that the present model can predict better than MEM for short fiber composites with the thermal conductivity ratio of 1,000 or higher and for $f$ of 0.4 or higher.

\section{Conclusions}

The final forms for the thermal conductivities of aligned short fiber composites in the longitudinal and transverse directions are semi-explicitly expressed as functions of constituents' thermal conductivities, fiber aspect ratio, and the aspect ratio of the matrix surrounding the fiber by using GSCM. The results are compared with the upper and lower bounds proposed by $\mathrm{Wu}$ to be validated. The present model is confirmed to be extended to composites with extreme shapes such as sphere and continuous fiber by simply changing the confocality variable. It can be concluded through the comparison of the analytical results by the present model and MEM and the experimental results that the present model predicts more accurately the longitudinal thermal conductivity of the short fiber composite than MEM, especially for the thermal conductivity ratio of 1,000 or higher and the fiber volume fraction of 0.4 or higher. 


\section{Acknowledgments}

This work was supported by research grants from the Catholic University of Daegu in 2010.

\section{REFERENCES}

1) R. Hill: J. Mech. Phys. Solids 13 (1965) 213-222.

2) B. Budiansky: J. Mech. Phys. Solids 13 (1965) 223-227.

3) J. D. Eshelby: Proc. R. Soc. Lond. A 241 (1957) 376-396.

4) T. Mori and K. Tanaka: Acta Metal. 21 (1973) 571-574.

5) M. Taya and T. W. Chou: Int. J. Solids Struct. 17 (1981) 553-563.

6) G. P. Tandon and G. J. Weng: Polymer Comp. 5 (1984) 327-333.

7) H. Hatta and M. Taya: Int. J. Eng. Sci. 24 (1986) 1159-1172.

8) H. Hatta, M. Taya, F. A. Kulacki and J. F. Harder: J. Comp. Mater. 26 (1992) 612-625.
9) H. Hatta and M. Taya: J. Appl. Phys. 58 (1985) 2478-2486.

10) E. H. Kerner: Proc. Phys. Soc. B 69 (1956) 802-807.

11) R. M. Christensen and K. H. Lo: J. Mech. Phys. Solids 27 (1979) 315330.

12) R. M. Christensen: J. Elasticity 50 (1998) 15-25

13) C. L. Tucker III and E. Liang: Comp. Sci. Tech. 59 (1999) 655-671.

14) D. P. H. Hasselman and L. F. Johnson: J. Comp. Mater. 21 (1987) 508515.

15) J. D. Felske: Int. J. Heat Mass Transfer 47 (2004) 3453-3461.

16) Y. M. Lee, R. B. Yang and S. S. Gau: Int. Comm. Heat Mass Transfer 33 (2006) 142-150.

17) T. Miloh and Y. Benveniste: J. Appl. Phys. 63 (1988) 789-796.

18) L. Wu: Int. J. Eng. Sci. 48 (2010) 417-428.

19) C. P. Wong and R. S. Bollampally: J. Appl. Polymer Sci. 74 (1999) 3396-3403.

20) J. K. Lee: Thermal Cycling and Creep Resistance of Metal Matrix Composites, (University of Washington, Seattle, 1996) pp. 89-94. 